\title{
Plpelle Prospective ENDOmetrial carcinoma (PIPENDO) study, pre-operative recognition of high risk endometrial carcinoma: a multicentre prospective cohort study
}

Nicole C. M. Visser ${ }^{1 *}$, Johan Bulten ${ }^{1}$, Anneke A. M. van der Wurff², Erik A. Boss ${ }^{3}$, Carolien M. Bronkhorst ${ }^{4}$, Harrie W. H. Feijen ${ }^{5}$, Joke E. Haartsen ${ }^{6}$, Hilde A. D. M. van Herk' ${ }^{7}$ Ineke M. de Kievit ${ }^{8}$, Paul J. J. M. Klinkhamer ${ }^{9}$, Brenda M. Pijlman ${ }^{10}$, Marc P. M. L. Snijders ${ }^{11}$, Ingrid Vandenput ${ }^{12}$, M. Caroline Vos ${ }^{13}$, Peter E. J. de Wit ${ }^{14}$, Lonneke V. van de Poll-Franse ${ }^{15,16}$, Leon F.A.G. Massuger ${ }^{17}$ and Johanna M. A. Pijnenborg ${ }^{18}$

\begin{abstract}
Background: Endometrial carcinoma is the most common gynaecologic malignancy in industrialised countries and the incidence is still rising. Primary treatment is based on preoperative risk classification and consists in most cases of hysterectomy with bilateral salpingo-oophorectomy. In patients with serous and clear cell histology a complete surgical staging is mandatory. However, in routine clinical practice final histology regularly does not correspond with the preoperative histological diagnosis. This results in both over and under treatment.
\end{abstract}

Methods/Design: The aim of this multicentre, prospective cohort study is to select a panel of prognostic biomarkers to improve preoperative diagnosis of endometrial carcinoma in order to identify those patients that need extended surgery and/or additional treatment. Additionally, we will determine whether incorporation of cervical cytology and comorbidity could improve this preoperative risk classification. All patients treated for endometrial carcinoma in the participating hospitals from September 2011 till December 2013 are included. Patient characteristics, as well as comorbidity are registered. Patients without preoperative histology, history of hysterectomy and/or endometrial carcinoma or no surgical treatment including hysterectomy are excluded. The preoperative histology and final pathology will be reviewed and compared by expert pathologists. Additional immunohistochemical analysis of IMP3, p53, ER, PR, MLH1, PTEN, beta-catenin, p16, Ki-67, stathmin, ARID1A and L1CAM will be performed. Preoperative histology will be compared with the final pathology results. Follow-up will be at least 24 months to determine risk factors for recurrence and outcome.

Discussion: This study is designed to improve surgical treatment of endometrial carcinoma patients. A total of 432 endometrial carcinoma patients were enrolled between 2011 and 2013. Follow-up will be completed in 2015. Preoperative histology will be evaluated systematically and background endometrium will be classified. This is the first study incorporating immunohistochemistry, cervical cytology and comorbidity to define the optimal panel of prognostic biomarkers that contribute in clinical decision making in the management of endometrial carcinoma.

Trial registration: Netherlands Trial Register number NTR3503

Keywords: Endometrial carcinoma, Histological diagnosis, Endometrial sampling, Postmenopausal bleeding, Observational cohort study, Risk assessment

\footnotetext{
* Correspondence: nicole.visser@radboudumc.nl

Deceased

'Dept. Pathology, Radboud university medical centre, P.O. Box 9101, 6500 HB Nijmegen, The Netherlands

Full list of author information is available at the end of the article
} 


\section{Background}

Endometrial carcinoma (EC) is the most common gynaecologic malignancy in the United States with approximately 52,630 diagnosed cases annually [1]. In the Netherlands the incidence is about 1900 women, with a mortality rate of 480 [2]. The incidence is still rising due to increased life expectancy and obesity as important risk factor [3]. Although the majority of patients are diagnosed at an early stage with a favourable prognosis, still around $20 \%$ of patients die from the disease [4]. ECs are staged according to the 2009 Fédération Internationale de Gynécologie et d'Obstétrique (FIGO) classification. ECs are divided into two types. The majority of ECs are classified as type I and are related to unopposed oestrogenic stimulation resulting from obesity or exogenous hormone use and originate from hyperplastic endometrium. This tumour type is associated with early stage disease, endometrioid histology, and a favourable outcome after surgery [5]. In contrast, type II carcinomas are unrelated to oestrogenic stimulation and arise in a background of atrophic endometrium. Type II carcinomas are associated with advanced stage, high grade, non-endometrioid histology, and an overall a poor prognosis [5]. A recent study suggests the existence of a third type of EC characterised by low grade endometrioid endometrial carcinoma (EEC) and a background of atrophic endometrium [6]. This third type of EC may have a poorer prognosis when compared to type I carcinomas [6]. However, recently published data of The Cancer Genome Atlas (TCGA) Research Network, identified four subgroups of EC based on molecular classifiers such as TP53, PTEN and microsatellite instability [7]. This supports the need for adjusting the currently used classification.

\section{Primary treatment}

Primary treatment is currently based on preoperative risk classification and consists of hysterectomy with bilateral salpingo-oophorectomy. In uterine papillary serous carcinoma (UPSC) and clear cell carcinoma (CCC) a complete surgical staging is mandatory because of the high risk of extra-uterine disease [8-10]. Although the presence of lymph node metastasis is an unfavourable predictor for disease specific survival, data of Kwon et al. demonstrated that high-risk uterine factors including high grade tumour type, deep myometrial invasion, and cervical stromal involvement are more significant determinants of survival in EC than pelvic-node status [11]. The current study focuses on diagnosis and preoperative risk assessment of patients with EC.

\section{Preoperative diagnosis}

During the last decades dilatation and curettage (D\&C) has been replaced by minimally invasive techniques for endometrial sampling in an outpatient setting. The amount of tissue obtained from endometrial sampling is relatively small and there can be different subtypes of $\mathrm{EC}$ in one tumour, making routine histological discrimination between EEC and a high grade, UPSC or CCC difficult. Moreover, in $30 \%$ the amount of tissue obtained with outpatient endometrial sampling is insufficient for diagnosis [12]. Previous studies found discrepancy percentages between 15 and $40 \%$, including both grade and histological subtype [13-17]. When preoperative diagnosis was based on $\mathrm{D} \& \mathrm{C}$ or endometrial sampling, a preoperative diagnosis of grade 1 was concordant with the final diagnosis in $85 \%$ of cases. However, high grade lesions were more frequently underestimated by endometrial sampling compared to $\mathrm{D} \& \mathrm{C}[18]$.

\section{Immunohistochemical analysis in preoperative endometrial sampling}

Identification of a panel of immunohistochemical (IHC) markers may be helpful to establish a reliable preoperative risk classification. A brief summary of the selected markers that will be tested is given in Table 1. P53 immunopositivity is associated with non-endometrioid EC [19]. Negative IHC for oestrogen and progesterone receptors can predict lymph node metastasis and is associated with decreased survival [20]. Double negative hormone receptor status and p53 immunopositivity correlates with lymph node metastasis, high FIGO stage, non-endometrioid histology, high grade and poor prognosis [20]. Insulin-like growth factor II messenger RNA-binding protein 3 (IMP3) is a foetal protein not expressed in normal adult tissues. This oncoprotein plays an important role in tumour growth, migration and invasion. IMP3 could contribute to the preoperative identification of type II tumours, since it is more frequently expressed in UPSC and CCC when compared to EEC (resp. $78 \%, 57 \%$ and $15 \%$ ) [21]. A recent study showed that L1CAM is the best predicting prognostic factor in FIGO stage I, type I EC and superior to the standard used multifactor risk score (myometrial invasion, tumour grade and lymph space or vascular invasion) [22]. L1CAM immunohistochemistry can improve the identification of patients at risk for recurrent disease. However, all the mentioned biomarkers are lacking validation on pre-operative histological samples and are based on singles studies. Further research has to validate these promising results.

\section{Preoperative diagnosis of EC in cervical cytology}

The presence of endometrial cells in cervical cytology in postmenopausal women is strongly associated with endometrial pathology [23]. Abnormal cervical cytology is associated with extra-uterine disease in patients with UPSC and with cervical involvement in patients with EEC [24]. A combination of preoperative cervical 
Table 1 Immunohistochemical analysis

\begin{tabular}{|c|c|c|c|}
\hline \multicolumn{2}{|c|}{ Immunohistochemical marker } & \multirow{2}{*}{$\begin{array}{l}\text { Results } \\
\text { IMP3 is more frequently expressed in UPSC and CCC than in EEC } \\
\text { (resp. } 78 \%, 57 \% \text { and } 15 \% \text { of the tumours were positive). }\end{array}$} & \multirow{2}{*}{$\begin{array}{l}\text { Ref. } \\
{[21]}\end{array}$} \\
\hline IMP3 & Insulin-like growth factor II mRNA-binding protein 3 & & \\
\hline P53 & & $\begin{array}{l}\text { P53 is more expressed in non-endometrioid endometrial } \\
\text { carcinomas than in EEC. Expression is also related to higher } \\
\text { tumour grade. }\end{array}$ & [19] \\
\hline$E R$ and $P R$ & Oestrogen and progesterone receptor & $\begin{array}{l}\text { Negative receptors were associated with lymph node metastasis } \\
\text { and decreased survival. ER and PR expression is lower in } \\
\text { non-endometrioid endometrial carcinomas than in EEC. }\end{array}$ & {$[20,34]$} \\
\hline MLH1 & MutL homolog 1 & $\begin{array}{l}\text { Loss of expression of mismatch repair proteins is seen in high } \\
\text { grade EEC and not in UPSC and CCC. Loss of MLH1 expression } \\
\text { is associated with longer survival. }\end{array}$ & {$[35,36]$} \\
\hline PTEN & Phosphatase and tensin homologue & PTEN positivity is more frequently found in UPSC than EEC. & [19] \\
\hline Beta-catenin & & $\begin{array}{l}\text { Positive beta-catenin expression is associated with decreased } \\
\text { stage, decreased grade and negative lymph node status }\end{array}$ & [37] \\
\hline P16 & & $\begin{array}{l}\text { Loss of p16 expression is significantly correlated with high FIGO } \\
\text { stage and serous and clear cell histological subtype. }\end{array}$ & [38] \\
\hline Ki-67 & & $\begin{array}{l}\text { Higher Ki-67 expression is associated with higher tumour grade. } \\
\text { UPSC and CCC show higher Ki-67 proliferation index than EEC. }\end{array}$ & [34] \\
\hline Stathmin & & $\begin{array}{l}\text { Stathmin overexpression was associated with non-endometrioid } \\
\text { histology, high grade and poor disease-specific survival. }\end{array}$ & [39] \\
\hline ARID1A & AT-rich interactive domain $1 \mathrm{~A}$ gene & $\begin{array}{l}\text { Loss of ARID1A expression is significantly more frequent in high } \\
\text { grade EEC compared to UPSC. }\end{array}$ & [40] \\
\hline L1CAM & L1 cell adhesion molecule & $\begin{array}{l}\text { L1CAM is associated with higher grade and non-endometrioid } \\
\text { histology. Moreover, L1CAM positive EC have statistical significant } \\
\text { poorer disease-free survival and overall survival. }\end{array}$ & [22] \\
\hline
\end{tabular}

$\overline{U P S C}=$ Uterine papillary serous carcinoma; $C C C=$ Clear Cell Carcinoma; $E E C=$ Endometrioid endometrial carcinoma; $E C=$ endometrial carcinoma; $F I G O=$ Fédération Internationale de Gynécologie et d'Obstétrique

cytology with endometrial sampling might better predict final histology and risk for extended disease. In a study of Kinde et al. DNA was extracted from cervical smears to detect genetic disorders present in EC [25]. The mutation profile found in the primary tumour was found in all of the cervical smears [25]. These results indicate that cervical cytology might be a reliable and minimal invasive source of material for detection of EC.

\section{Comorbidity and EC}

The impact of comorbidity on cancer outcome has been underestimated for a long time. Recently published data demonstrated that EC patients with cardiovascular disease, previous malignancy and diabetes have a significantly decreased survival of 15-17 \% compared to patients without comorbidity [26]. Additionally, patients with diabetes and EC have more comorbidities, higher body mass index (BMI) and higher FIGO stage, compared to those without diabetes [27]. There is also a significant increase in the risk of EC-specific mortality among women with diabetes [28]. Although obesity is a risk factor for development of EC, obesity seems not related to overall survival [29]. Yet, comorbidity has demonstrated to influence the outcome in EC [30].

In summary, the main challenging issue concerning clinical management of EC patients is underscored by the discordance between the preoperative risk classification of the tumour and the final surgical pathology. At the moment a subgroup of patients needs either a secondary surgical staging procedure or additional chemotherapy and/or radiation therapy. With the current study we want to select a panel of the most accurate biomarkers that can be used in daily practice for preoperative diagnosis of EC. This will aid in improving the concordance between preoperative and final histological diagnosis and thus prevent over and under treatment. Incorporating cervical cytology and comorbidity could potentially improve a proper risk classification in EC patients.

\section{Methods/Design \\ Objective \\ Primary objective}

To determine whether standardized evaluation of endometrial biopsies with additional immunohistochemical analysis, could predict final histological type, tumour grade and stage.

\section{Secondary objective}

To determine whether additional immunohistochemical analysis on endometrial biopsies could predict recurrence and disease free survival. Additionally, to determine 
whether incorporation of abnormal cervical cytology and comorbidity attributes to an improved risk classification.

\section{Study design}

Multicentre, prospective cohort study in nine hospitals in the Netherlands. From September 1st 2011 till December 1st 2013 all patients treated for EC in participating hospitals are included. Patients without preoperative histology, history of hysterectomy and/or endometrial carcinoma or no surgical treatment including hysterectomy are excluded. Patient characteristics, as well as comorbidity (Charlson index), BMI, family history of hereditary syndromes (BRCA1/2, Lynch syndrome), postmenopausal status and parity are registered. Based on the present comorbidities, all patients are assigned a comorbidity score based on the Age-Adjusted Comorbidityindex as described by Charlson et al. [31], with EC being excluded from the scoring. Treatment and final pathological diagnosis are registered as well as occurrence of recurrent disease during at least 24 months follow-up.

\section{Tissue specimens}

The endometrial biopsy or curettage on which the diagnosis of EC was made will be used for systematic evaluation by pathologists with special interest in gynaecologic pathology. Additional IHC analysis of IMP3, p53, ER, PR, MLH1, PTEN, beta-catenin, p16, Ki-67, stathmin, ARID1A and L1CAM will be performed (Table 1). Final pathology will be reviewed by the expert pathologists and compared with the preoperative histological diagnosis. The pathologists will be blinded for clinicopathological information and outcome.

\section{Methods}

Tissue specimens are collected centrally at the department of Pathology, Radboud university medical centre in Nijmegen. Pre-operative samples will be evaluated on the amount of tissue (quantitatively and qualitatively), the presence of hyperplasia, atypia, endometrial intraepithelial carcinoma (EIC), invasive growth, background endometrium, tumour percentage and tumour type and grade. IHC staining will be performed on formalin-fixed, paraffin-embedded tissue of the pre-operative samples. IHC staining will be graded semiquantitatively by considering the percentage and intensity of the staining. A staining index will be calculated as the product of staining intensity and staining area.

\section{Statistical analysis}

For the primary objective, results of endometrial biopsy and curettage will be compared with final pathology results. Both univariate as well as multivariate analysis will be performed to determine whether immunohistochemical markers contribute to prediction of final pathology.
For the secondary objective we will also include abnormal cervical cytology and the Age-Adjusted Comorbidity-index as factors in univariate and multivariate analysis. In order to determine risk factors for recurrence Kaplan-Meier survival curves will be calculated to determine outcome after a follow-up time of 24 months. Statistical analysis will be performed using the Statistical and Data management package SPSS 20.0.

\section{Sample size calculation}

Calculation of the sample size is based on the primary outcome variable of the study, which is high risk endometrial carcinoma. The smallest outcome group, in this case patients with high risk endometrial carcinoma, should be $10-20$ times the amount of independent variables used.

Independent variables in the analyses will be: age (dichotomous), grade (1, 2 and 3 ) and the best predictive immunohistochemical markers. For the sample size calculation we assume to include six immunohistochemical markers in the multivariate analysis. Grade count as two variables because we use it as a trichotomous variable, which makes the total variables nine.

Table 2 Clinicopathological characteristics of 432 women with endometrial carcinoma. Values are presented as median (range) or number (\%)

\begin{tabular}{|c|c|c|}
\hline \multicolumn{3}{|l|}{ Characteristics } \\
\hline Age at primary surgery, years & 66 & $(41-90)$ \\
\hline \multicolumn{3}{|l|}{ Pre-operative histology ${ }^{a}$} \\
\hline Office endometrial biopsy & 311 & $(72.0)$ \\
\hline Hysteroscopic biopsy & 128 & $(29.6)$ \\
\hline Curettage & 75 & $(17.4)$ \\
\hline \multicolumn{3}{|l|}{ Histological subtype ${ }^{\text {b }}$} \\
\hline Endometrioid & 370 & $(85.6)$ \\
\hline Serous papillary & 33 & (7.6) \\
\hline Clear cell & 9 & $(2.1)$ \\
\hline Mucinous adenocarcinoma & 2 & $(0.5)$ \\
\hline Carcinosarcoma & 18 & $(4.2)$ \\
\hline \multicolumn{3}{|l|}{ Histological grade ${ }^{b}$} \\
\hline 1 & 193 & $(44.7)$ \\
\hline 2 & 127 & $(29.4)$ \\
\hline 3 & 112 & $(25.9)$ \\
\hline \multicolumn{3}{|l|}{ FIGO 2009 stage } \\
\hline I & 362 & $(83.8)$ \\
\hline ॥ & 27 & $(6.3)$ \\
\hline III & 35 & $(8.1)$ \\
\hline IV & 8 & (1.9) \\
\hline
\end{tabular}

a 82 patients have more than one pre-operative histological sample ${ }^{\mathrm{b}}$ Unrevised classification based on hysterectomy specimen 
The amount of subjects in the smallest group therefore should lie between 90 and 180. However, as a rule of thumb, the amount of subjects should never be lower than 100 .

With an expected high risk endometrial carcinoma rate of $25 \%$ at least 400 patients with endometrial carcinoma should be included to include at least $100 \mathrm{pa}$ tients with high risk endometrial carcinoma.

\section{Ethical considerations}

This study is approved by the local medical ethical committee of the St Elisabeth Hospital Tilburg. According to the protocol "Code for Proper Use of Human Tissue", all collected patient material will be coded, and patient name and date of birth are not entered into the database (Dutch Federation for Biomedical Scientific Societies, www.federa.org). We did not obtain written informed consent from patients because we use data anonymously according to the "Code for Proper Use of Human Tissue". Included patients are informed about tissue and data use for scientific purpose in general and made no drawbacks.

\section{Discussion}

A total of 432 EC patients from nine hospitals were collected between September 2011 and December 2013. The inclusion of patients has finished and we are now analysing the data. Follow-up will be completed in December 2015. The various histological subtypes of EC are all represented in this study group with $86 \%$ EEC, $8 \%$ UPSC and $2 \%$ CCC based on hysterectomy evaluation. This is in line with percentages reported in the Netherlands Cancer Registry [32]. Clinicopathological characteristics are shown in Table 2. Atypical hyperplasia is diagnosed in $13 \%$ of the preoperative endometrial samples, where final diagnosis on hysterectomy was EC.

We will determine if a diagnostic panel of IHC markers can improve the preoperative diagnosis for risk selection. This is the first study combining L1CAM with other markers to find the optimal panel of biomarkers for the preoperative diagnosis of EC. The additional value of immunohistochemical analysis in $\mathrm{EC}$ has been demonstrated in the large multicentre MoMaTEC trial [20], yet, this study focussed on predicting lymph node metastasis and prognosis in relation to treatment. Our focus is on preoperative risk classification with respect to histological type and tumour grade. Preoperative and final surgical pathology will be revised by expert pathologists. Furthermore, preoperative biopsy and curettage will be evaluated systematically, and compared with final histology. Additionally, background endometrium will be classified as: atrophic endometrium, hyperplastic endometrium, normal proliferative endometrium or indeterminate. Since, in a previous study on hysterectomy specimens, atrophic background endometrium was found to be an independent prognostic factor for patients with grade 1 EEC [6]. To date, no studies on the prognostic value of background endometrium in preoperative endometrial sampling are published.

Due to tumour heterogeneity and focal staining patterns, IHC on endometrial biopsies may not always be representative for the whole tumour. Most studies on IHC in endometrial carcinomas were performed on hysterectomy specimens. Yet, our clinical challenge is to select high risk tumour preoperatively on a limited amount of material. Our study design represents daily practice and with this study we will determine whether additional IHC analysis could predict final histology. Huang et al. reported comparable sensitivity for detecting high grade EC with Pipelle versus curettage [33]. The predictive value between endometrial biopsies and curettage might be different when IHC is applied, and hence influence outcome. To date, there are no studies on the influence of IHC on the difference in predictive value between biopsy and curettage. By using IHC the difference in the amount of material collected by biopsy and curettage might become relevant. Interestingly, incorporation of comorbidity in the preoperative risk classification has not been studied so far.

Summarizing, systematic preoperative evaluation of both tumour and patient characteristics could give maximal information and result in patient tailored treatment in patients with EC.

\section{Abbreviations \\ CCC: Clear cell carcinoma; D\&C: Dilatation and curettage; EC: Endometrial carcinoma; EEC: Endometrioid endometrial carcinoma; EIC: Endometrial intraepithelial carcinoma; FIGO: Fédération Internationale de Gynécologie et d'Obstétrique; IHC: Immunohistochemical; UPSC: Uterine papillary serous carcinoma.}

\section{Competing interests}

The authors declare that they have no competing interests.

\section{Authors' contributions}

$J P, N V, A W, J B, L P, L M$ were involved in conception and design of the study. $E B, C B, J B, H F, J H, H H, I K, P K, B P, M S, I V, M V, P W$ and AW assisted in data collection. NV and JP drafted the first manuscript. All authors edited the manuscript and read and approved the final draft.

\section{Acknowledgements}

This study is supported in part by a grand from the Ruby and Rose Foundation, The Netherlands.

\section{Author details}

${ }^{1}$ Dept. Pathology, Radboud university medical centre, P.O. Box 9101, 6500 HB Nijmegen, The Netherlands. ${ }^{2}$ Dept. Pathology, St Elisabeth Hospital, Tilburg, The Netherlands. ${ }^{3}$ Dept. Obstetrics and Gynaecology, Maxima Medical Centre, Veldhoven and Eindhoven, The Netherlands. ${ }^{4}$ Dept. Pathology, Jeroen Bosch Hospital, 's-Hertogenbosch, The Netherlands. ${ }^{5}$ Dept. Obstetrics and Gynaecology, Amphia Hospital, Breda, The Netherlands. ${ }^{6}$ Dept. Obstetrics and Gynaecology, Elkerliek Hospital, Helmond, The Netherlands. ${ }^{7}$ Dept. Pathology, Elkerliek Hospital, Helmond, The Netherlands. ${ }^{8}$ Dept. Pathology, Canisius Wilhemina Hospital, Nijmegen, The Netherlands. 'Dept. Pathology, PAMM, Eindhoven, The Netherlands. ${ }^{10}$ Dept. Obstetrics and Gynaecology, Jeroen Bosch Hospital, 's-Hertogenbosch, The Netherlands. ${ }^{11}$ Dept. Obstetrics 
and Gynaecology, Canisius Wilhemina Hospital, Nijmegen, The Netherlands. ${ }^{12}$ Dept. Obstetrics and Gynaecology, Catharina Hospital, Eindhoven, The Netherlands. ${ }^{13}$ Dept. Obstetrics and Gynaecology, St Elisabeth Hospital, Tilburg, The Netherlands. ${ }^{14}$ Dept. Pathology, Amphia Hospital, Breda, The Netherlands. ${ }^{15}$ Dept. of Medical and Clinical Psychology, Tilburg University, Tilburg, The Netherlands. ${ }^{16}$ Comprehensive Cancer Centre the Netherlands, Eindhoven, The Netherlands. ${ }^{17}$ Dept. Obstetrics and Gynaecology, Radboud university medical centre, Nijmegen, The Netherlands. ${ }^{18}$ Dept. Obstetrics and Gynaecology, TweeSteden Hospital, Tilburg, The Netherlands.

Received: 7 November 2014 Accepted: 10 June 2015

Published online: 30 June 2015

\section{References}

1. Siegel R, Ma J, Zou Z, Jemal A. Cancer statistics, 2014. CA Cancer J Clin. 2014;64(1):9-29.

2. Netherlands Cancer Registry. [http://www.cijfersoverkanker.nl]

3. Boll D, Karim-Kos HE, Verhoeven RH, Burger CW, Coebergh JW, van de PollFranse LV, et al. Increased incidence and improved survival in endometrioid endometrial cancer diagnosed since 1989 in The Netherlands: a population based study. Eur J Obstet Gynecol Reprod Biol. 2013;166(2):209-14.

4. Creasman WT, Odicino F, Maisonneuve P, Quinn MA, Beller U, Benedet JL, et al. Carcinoma of the corpus uteri. FIGO 26th annual report on the results of treatment in gynecological cancer. Int J Gynaecol Obstet Off Organ Int Fed Gynaecol Obstet. 2006;95 Suppl 1:S105-43.

5. Bokhman JV. Two pathogenetic types of endometrial carcinoma. Gynecol Oncol. 1983;15(1):10-7.

6. Geels YP, Pijnenborg JM, van den Berg-van Erp SH, Bulten J, Visscher DW, Dowdy SC, et al. Endometrioid endometrial carcinoma with atrophic endometrium and poor prognosis. Obstet Gynecol. 2012;120(5):1124-31.

7. Cancer Genome Atlas Research N, Kandoth C, Schultz N, Cherniack AD Akbani R, Liu Y, et al. Integrated genomic characterization of endometrial carcinoma. Nature. 2013:497(7447):67-73.

8. Oncoline: cancer clinical practice guidelines. [www.oncoline.nl]

9. Boruta 2nd DM, Gehrig PA, Fader AN, Olawaiye AB. Management of women with uterine papillary serous cancer: a Society of Gynecologic Oncology (SGO) review. Gynecol Oncol. 2009;115(1):142-53.

10. Olawaiye AB, Boruta 2nd DM. Management of women with clear cell endometrial cancer: a Society of Gynecologic Oncology (SGO) review. Gynecol Oncol. 2009;113(2):277-83.

11. Kwon JS, Qiu F, Saskin R, Carey MS. Are uterine risk factors more important than nodal status in predicting survival in endometrial cancer? Obstet Gynecol. 2009;114(4):736-43.

12. Visser NC, Breijer MC, Herman MC, Bekkers RL, Veersema S, Opmeer BC, et al. Factors attributing to the failure of endometrial sampling in women with postmenopausal bleeding. Acta Obstet Gynecol Scand. 2013;92(10):1216-22.

13. Petersen RW, Quinlivan JA, Casper GR, Nicklin JL. Endometrial adenocarcinoma - presenting pathology is a poor guide to surgical management. Aust N Z J Obstet Gynaecol. 2000;40(2):191-4.

14. Frumovitz $M$, Singh DK, Meyer $L$, Smith DH, Wertheim I, Resnik E, et al. Predictors of final histology in patients with endometrial cancer. Gynecol Oncol. 2004;95(3):463-8.

15. Leitao Jr MM, Kehoe S, Barakat RR, Alektiar K, Gattoc LP, Rabbitt C, et al. Accuracy of preoperative endometrial sampling diagnosis of $\mathrm{FIGO}$ grade 1 endometrial adenocarcinoma. Gynecol Oncol. 2008;111(2):244-8.

16. Neubauer NL, Havrilesky $\sqcup$, Calingaert B, Bulusu A, Bernardini MQ, Fleming ND, et al. The role of lymphadenectomy in the management of preoperative grade 1 endometrial carcinoma. Gynecol Oncol. 2009;112(3):511-6.

17. Helpman L, Kupets R, Covens A, Saad RS, Khalifa MA, Ismiil N, et al. Assessment of endometrial sampling as a predictor of final surgical pathology in endometrial cancer. Br J Cancer. 2014;110(3):609-15.

18. Leitao Jr MM, Kehoe S, Barakat RR, Alektiar K, Gattoc LP, Rabbitt C, et al. Comparison of D\&C and office endometrial biopsy accuracy in patients with FIGO grade 1 endometrial adenocarcinoma. Gynecol Oncol. 2009;113(1):105-8.

19. Mhawech-Fauceglia P, Herrmann FR, Rai H, Tchabo N, Lele S, Izevbaye I, et al. IMP3 distinguishes uterine serous carcinoma from endometrial endometrioid adenocarcinoma. Am J Clin Pathol. 2010;133(6):899-908.

20. Trovik J, Wik E, Werner HM, Krakstad C, Helland H, Vandenput I, et al Hormone receptor loss in endometrial carcinoma curettage predicts lymph node metastasis and poor outcome in prospective multicentre trial. Eur J Cancer. 2013:49(16):3431-41.
21. Mhawech-Fauceglia P, Yan L, Liu S, Pejovic T. ER+ /PR+ /TFF3+/IMP3 immunoprofile distinguishes endometrioid from serous and clear cell carcinomas of the endometrium: a study of 401 cases. Histopathology. 2013;62(7):976-85

22. Zeimet AG, Reimer D, Huszar M, Winterhoff B, Puistola U, Azim SA, et al. L1CAM in early-stage type I endometrial cancer: results of a large multicenter evaluation. J Natl Cancer Inst. 2013;105(15):1142-50.

23. Siebers $A G$, Verbeek $A L$, Massuger LF, Grefte JM, Bulten J. Normal appearing endometrial cells in cervical smears of asymptomatic postmenopausal women have predictive value for significant endometrial pathology. Int J Gynecol Cancer. 2006;16(3):1069-74.

24. Roelofsen T, Geels YP, Pijnenborg JM, van Ham MA, Zomer SF, van Tilburg JM, et al. Cervical cytology in serous and endometrioid endometrial cancer. Int J Gynecol Pathol. 2013;32(4):390-8.

25. Kinde I, Bettegowda C, Wang Y, Wu J, Agrawal N, Shih le M, et al. Evaluation of DNA from the Papanicolaou test to detect ovarian and endometrial cancers. Sci Transl Med. 2013;5(167):167ra164.

26. Boll D, Verhoeven RH, van der Aa MA, Lybeert ML, Coebergh JW, JanssenHeijnen ML. Adherence to national guidelines for treatment and outcome of endometrial cancer stage I in relation to co-morbidity in southern Netherlands 1995-2008. Eur J Cancer. 2011;47(10):1504-10.

27. Zanders $M M$, Boll $D$, van Steenbergen $L N$, van de Poll-Franse LV, Haak HR. Effect of diabetes on endometrial cancer recurrence and survival. Maturitas. 2013;74(1):37-43.

28. Liao C, Zhang D, Mungo C, Tompkins DA, Zeidan AM. Is diabetes mellitus associated with increased incidence and disease-specific mortality in endometrial cancer? A systematic review and meta-analysis of cohort studies. Gynecol Oncol. 2014;135(1):163-71.

29. Nevadunsky NS, Van Arsdale A, Strickler HD, Moadel A, Kaur G, Levitt J, et al. Obesity and age at diagnosis of endometrial cancer. Obstet Gynecol. 2014;124(2 Pt 1):300-6.

30. Robbins JR, Gayar OH, Zaki M, Mahan M, Buekers T, Elshaikh MA. Impact of age-adjusted Charlson comorbidity score on outcomes for patients with early-stage endometrial cancer. Gynecol Oncol. 2013;131(3):593-7.

31. Charlson M, Szatrowski TP, Peterson J, Gold J. Validation of a combined comorbidity index. J Clin Epidemiol. 1994;47(11):1245-51.

32. Boll D, Verhoeven $\mathrm{RH}$, van der Aa MA, Pauwels P, Karim-Kos HE, Coebergh JW et al. Incidence and survival trends of uncommon corpus uteri malignancies in the Netherlands, 1989-2008. Int J Gynecol Cancer. 2012;22(4):599-606.

33. Huang GS, Gebb JS, Einstein MH, Shahabi S, Novetsky AP, Goldberg GL. Accuracy of preoperative endometrial sampling for the detection of highgrade endometrial tumors. Am J Obstet Gynecol. 2007;196(3):243. e241-245.

34. Lax SF, Pizer ES, Ronnett BM, Kurman RJ. Clear cell carcinoma of the endometrium is characterized by a distinctive profile of p53, Ki-67, estrogen, and progesterone receptor expression. Hum Pathol. 1998;29(6):551-8.

35. Nelson GS, Pink A, Lee S, Han G, Morris D, Ogilvie T, et al. MMR deficiency is common in high-grade endometrioid carcinomas and is associated with an unfavorable outcome. Gynecol Oncol. 2013;131(2):309-14.

36. Peiro G, Diebold J, Mayr D, Baretton GB, Kimmig R, Schmidt M, et al. Prognostic relevance of hMLH1, hMSH2, and BAX protein expression in endometrial carcinoma. Mod Pathol. 2001;14(8):777-83.

37. Athanassiadou P, Athanassiades P, Grapsa D, Gonidi M, Athanassiadou AM, Stamati PN, et al. The prognostic value of PTEN, p53, and beta-catenin in endometrial carcinoma: a prospective immunocytochemical study. Int J Gynecol Cancer. 2007;17(3):697-704.

38. Engelsen IB, Stefansson I, Akslen LA, Salvesen HB. Pathologic expression of p53 or p16 in preoperative curettage specimens identifies high-risk endometrial carcinomas. Am J Obstet Gynecol. 2006;195(4):979-86.

39. Trovik J, Wik E, Stefansson IM, Marcickiewicz J, Tingulstad S, Staff AC, et al. Stathmin overexpression identifies high-risk patients and lymph node metastasis in endometrial cancer. Clin Cancer Res. 2011;17(10):3368-77.

40. Allo G, Bernardini MQ, Wu RC, Shih le M, Kalloger S, Pollett A, et al. ARID1A loss correlates with mismatch repair deficiency and intact p53 expression in high-grade endometrial carcinomas. Mod Pathol. 2014;27(2):255-61. 